\title{
Preparation and Characterization of Nanocrystalline h-BN Films Prepared by PECVD Method
}

\author{
J. Vilcarromero*, M.N.P. Carreño, I. Pereyra, \\ GNMD, PSI, EPUSP, University of São Paulo, \\ CEP 0524-970, P.O Box 61548, São Paulo, Brazil \\ N. C. Cruz, and E.C. Rangel \\ LPA, DFQ, Faculdade de Engenharia, Unesp-Guaratingueta \\ CEP 12516-410, São Paulo, Brazil
}

Received on 23 April, 2001

\begin{abstract}
This work describes a systematic study of the preparation of nano-crystalline thin h-BN films by Plasma-Enhanced Chemical Vapor Deposition (PECVD) technique. The samples were prepared at low temperatures using $\mathrm{B}_{2} \mathrm{H}_{6}$ and $\mathrm{N}_{2}$ as gas precursors. It is shown that the flow ratio among these gases has an important influence on the size of the crystallites (deduced by Raman spectroscopy). The $\mathrm{B}_{2} \mathrm{H}_{6} / \mathrm{N}_{2}$ flow ratio was varied from $1,6 \times 10^{-2}$ to $6.7 \times 10^{-4}$ leading to films presenting a crystallite size, which varied from $90 \mathrm{~nm}$ to amorphous, respectively. The XRD spectra show two peaks at 2 values around $42^{\circ}$ and $44^{\circ}$, which are associated to $<110>$ and $<100>$ h-BN crystalline directions, indicating, a $<002>$ preferential orientation for the $\mathrm{h}-\mathrm{BN}$ crystallites. The thermomechanical properties, as stress, hardness, and Young modulus were also studied and correlated with the structural properties. The composition of the films was obtained by RBS and EDS indicating Boron to Nitrogen ratio, close to stoichiometry for all the studied deposition conditions.
\end{abstract}

\section{Introduction}

In analogy to the carbon system, $\mathrm{BN}$ can exist in different crystallographic phases, among them the hexagonal and the cubic modifications; h-BN and c-BN have received great attention in view of coating applications. Like graphite h-BN consists of $\mathrm{sp}^{2}$-bonded hexagons. In h-BN, the boron and nitrogen atoms alternate in sequence of hexagonal layers. Sub modifications with higher disorder in the layer sequence are possible. Nevertheless, the h-BN has been considered as one of the most promising fine ceramics due to its high electrical resistivity, excellent thermal conductivity and extremely low dielectric constant [1-7]. It has also been developed as a potential alternative for fiber coatings in ceramic matrix composites on account of its lubrication and corrosion-resistant characteristics [7].

Different PVD (physical vapor deposition) and CVD (chemical vapor deposition) deposition methods to prepare $\mathrm{BN}$ have been reported, normally in temperatures above $600{ }^{\circ} \mathrm{C}[8-11]$. To prepare $\mathrm{BN}$ at low temperature is advantageous, however depending on the final thickness the films present structural instability related to internal stress. In this way the knowledge of the mechanical properties of these materials, such as stress, hardness, elastic modulus, and thermal expansion coefficient is of great interest. It is well known that the thermo mechanical properties of thin films are strongly correlated with the film structure such as defects, voids, network strain and the mean coordination number. In this paper, we present a systematic study of the influence of the substrate temperature and diborane to nitrogen flow rate on the structural and thermo mechanical properties of the hexagonal boron nitride thin films prepared by PECVD method at low temperatures (below $400{ }^{\circ} \mathrm{C}$ ).

\section{Experimental}

The Boron Nitride thin films were prepared using a standard $13.56 \mathrm{MHz}$ radio frequency PECVD reactor, capactively coupled, and utilizing different mixtures of $\left(\mathrm{B}_{2} \mathrm{H}_{6} / \mathrm{N}_{2}\right)$ as gas precursors. The set-up has been described in detail elsewhere [12, 13]. The rf power, substrate temperature, diborane to nitrogen flow rate, and the total pressure were systematically varied from one deposition to the other in order to analyze the effect of these parameters on the films properties.

Rutherford backscattering (RBS), X-ray photoelectron spectroscopes (XPS), and Energy Dispersive X-ray (EDS) spectroscopy and Elastic Recoil detect analyses (ERDA) were used to determine the chemical composition of the samples. In addition, XPS spectra were performed to analyze the short structural configuration of the h-BN films. The infrared absorption spectra were 
obtained by FTIR (in a Bio-Rad spectrometer) in films deposited onto c-Si substrates, in the $200-4000 \mathrm{~cm}^{-1}$ wave-number range. The micro-Raman spectra were obtained with a Renishaw spectrometer in backscattering configuration, at room temperature using the 514.5$\mathrm{nm}$ line of argon laser and X50 amplifier microscope. XRD measurements were performed in a Philips spectrometer using a $\mathrm{Cu}$ source in a low angle system to confirm the nano-crystalline phase.

Stress measurements were taken from films deposited onto $3 \times 25 \times 0.4 \mathrm{~mm}^{3}$ (111)-Si bars using a Sloan profilometer to determine the radius of curvature of the film-substrate composite. The stress was then calculated by the Stoney equation [14]:

$$
\sigma=[E /(1-\nu)]\left(t^{2} / 6 d R\right)
$$

where E, $\nu$ and $\mathrm{t}$ are the Young's modulus, Poisson's ratio, and thickness of the substrate, $\mathrm{R}$ and $\mathrm{d}$ are the radius of curvature and the thickness of the film, respectively. The hardness and Young Modulus were obtained in a Nanohardness system at different steps of measurements.

\section{Results}

The compositional analyses indicate that the produced films are close to stoichiometry with the $\mathrm{B} / \mathrm{N}$ ratio varying from 1 to 1.15 depending on sample preparation and experimental conditions. The concentration of carbon, oxygen and other elements found as contaminants was low but increase for samples prepared with low substrate temperature. Both molecular hydrogen and hydrogen bonded to boron and nitrogen increase when the substrate temperatures decrease as observed by ERDA and infrared spectra, respectively. The deposition rate varied from $2 \times 10^{-2}$ to $3.3 \times 10^{-1} \mathrm{~A} / \mathrm{s}$ and the sample thickness from 0.35 to $0.8 \mu \mathrm{m}$.

The XPS spectra for h-BN thin films prepared keeping all the deposition parameters constant and varying the substrate temperature are presented in Fig. 1. The hexagonal phase of boron nitride compound presents the B 1s core level at $190.5 \mathrm{eV}$ [15]. All our BN samples present a B 1s-core level at $190.3 \mathrm{eV}$ very close to the hexagonal BN phase. However, the samples prepared at room temperature present a low shoulder band at $192 \mathrm{eV}$, which is associated to boron oxide [15], in accordance to composition analyses, which indicates a higher presence of oxygen, compared to samples prepared at $340{ }^{\circ} \mathrm{C}$. In Fig. 2, the infrared spectra for the same samples presented at figure 1 are shown. The absorption band observed around $780 \mathrm{~cm}^{-1}$ and 1380 $\mathrm{cm}^{-1}$ are related to the BN hexagonal phase [16] and the absorption bands centered at $2450 \mathrm{~cm}^{-1}$ and 3250 $\mathrm{cm}^{-1}$ are related to hydrogen bonded to boron and nitrogen, respectively. The FTIR analysis as a function of the deposition temperature shows that for higher temperatures the hexagonal crystallite size, as indicated by the FWHM of the h-BN absorption band at $1380 \mathrm{~cm}^{-1}$, increases. For lower temperatures the hydrogen content of the films increases as indicated by the $\mathrm{N}-\mathrm{H}$ stretching band at $3250 \mathrm{~cm}^{-1}$. The deposition rate also increased for decreasing temperatures, varying from $9 \times 10^{-3}$ to $3.3 \times 10^{-1} \mathrm{~A} / \mathrm{s}$. The maximum sample thickness attained for 6 hrs deposition was $0.8 \mu \mathrm{m}$.

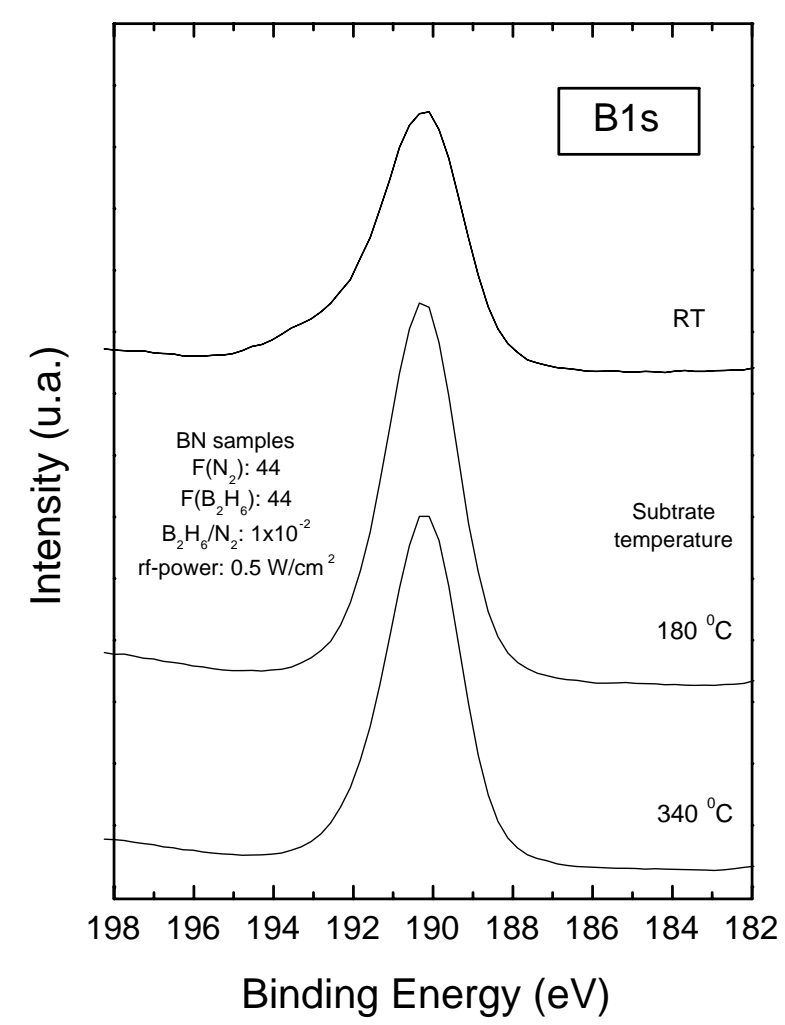

Figure 1. XPS spectra, showing the Boron 1s core level for samples grown at different substrate temperatures.

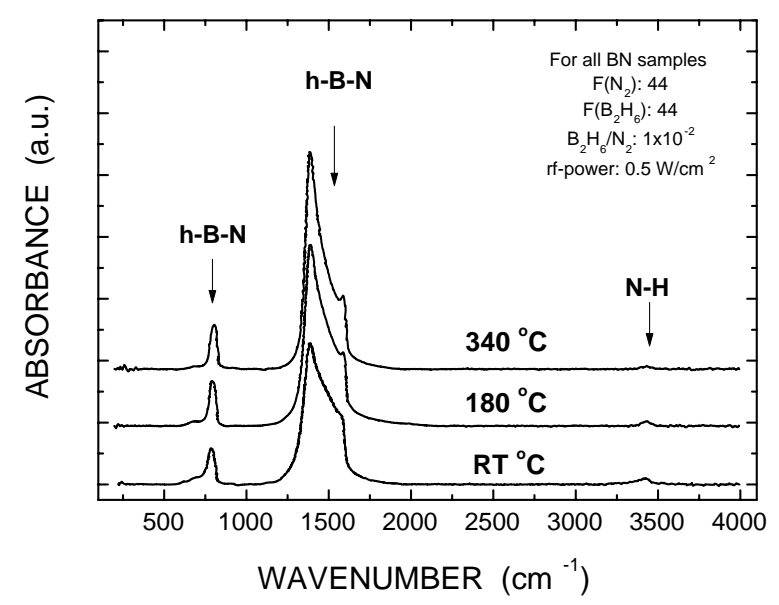

Figure 2. FTIR spectra for BN samples prepared at different substrate temperatures. 
The average grain size of the hexagonal crystallites was also estimated through Raman Spectroscopy. The Raman spectra of hexagonal BN samples present an absorption band in $1380 \mathrm{~cm}^{-1}$, according to the study by Nemanich et. al. [17] it is possible to estimate the average grain size of the nano-crystals present in the samples using the following relation:

$$
\Gamma_{1 / 2}=\frac{1447}{L_{a}}+8.70
$$

Where $\Gamma_{1 / 2}$ is a FWHM of the Lorentzian curve fitting the absorption band and $\mathrm{L}_{a}$ represents the equivalent grain size of the nano-crystals present in the sample. Fig. 3 presents the results obtained applying this equation to samples prepared at the same condition but changing the diborane flow. As it can be observed, the estimated average grain size of the h-BN thin film increases when the $\mathrm{B}_{2} \mathrm{H}_{6}$ flow increases. In the inset the XRD spectra for the sample with $50 \AA$ of crystalline size is shown and peaks for 42.3 and 44,3 degrees, associated to the $<100>$ and $<101>$ directions of BN hexagonal phase are evident.

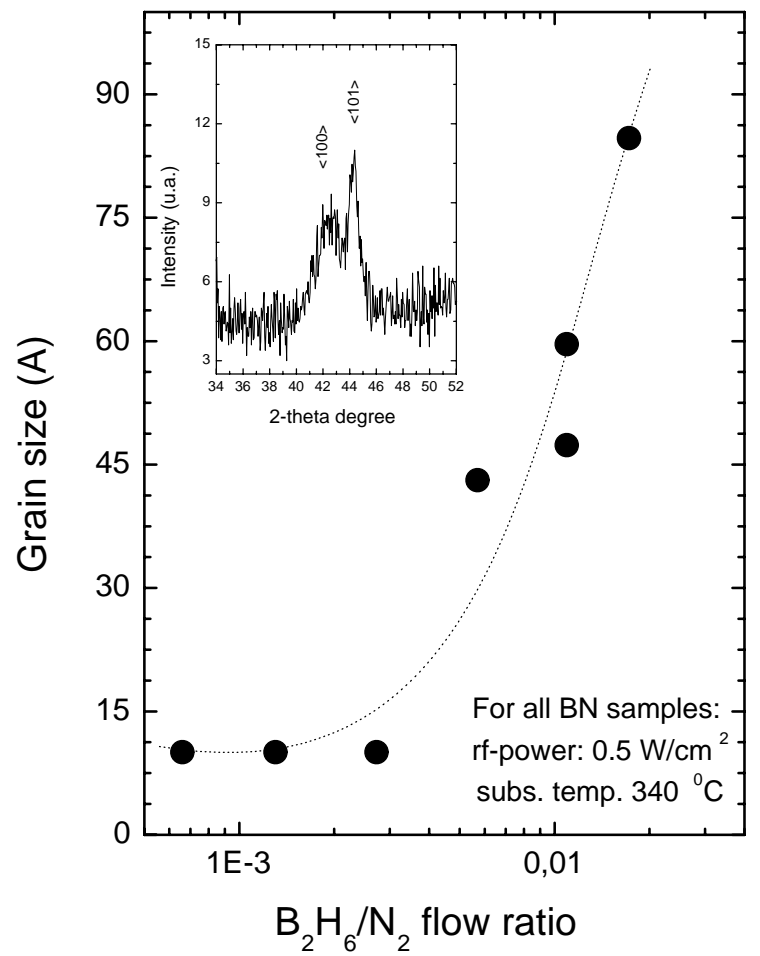

Figure 3. Grain size as a function of the substrate temperature of the $\mathrm{BN}$ samples obtained using the equation 2. In inset a XRD spectra of the equivalent $50 \AA$ of grain size.

The results for the intrinsic stress for the BN samples are shown in Fig. 4 (b), as it can be observed, the stress presents a minimum for substrate temperature around $180{ }^{\circ} \mathrm{C}$. The hardness and Young Modulus for the same hexagonal BN samples are shown in Fig. 4(a). The hardness is bellow 4 Gpa and presents an opposite behavior compared to the stress as the substrate temperature increases. In contrast, the Young modulus does not present an evident correlation with the substrate temperature.

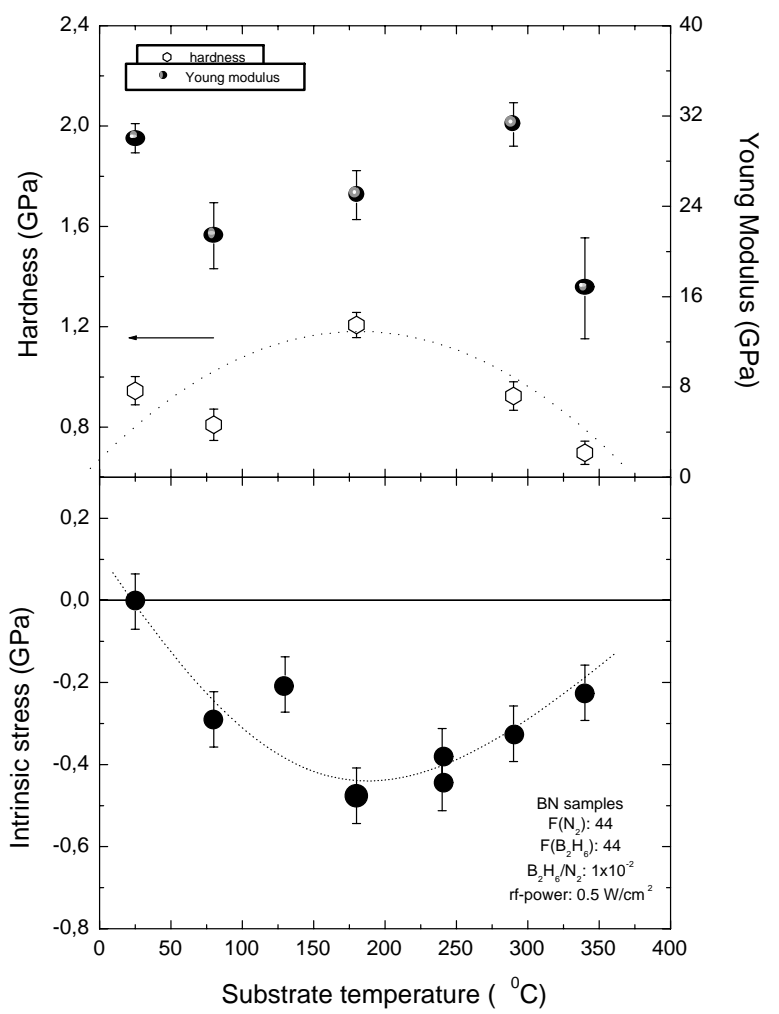

Figure 4. (a) Hardness and Young Modulus as function of substrate temperatures for the BN samples, and (b) BN samples intrinsic stress as function of the substrate temperature.

\section{Discussion.}

Infrared absorption spectroscopy is the most widely used method for BN films structural characterization since the hybridization states, $\mathrm{sp}^{2}$ or $\mathrm{sp}^{3}$, of the $\mathrm{B}-$ $\mathrm{N}$ bond are easily distinguished through well-identified absorption's bands. The h-BN and turbostatic phases are characterized by two infrared active TO phonons, giving a strong and asymmetrical absorption band $\mathrm{E}_{1 u}$ at about $1380 \mathrm{~cm}^{-1}$ attributed to in-plane stretching and a weaker band $A_{2 u}$ near $800 \mathrm{~cm}^{-1}$ resulting from an out-of plane B-N-B bending mode [16]. Thus the results shown in figure 2 combined with those in figures 1 and 3 indicate that it is also possible to grow Hexagonal BN samples with good structure and large grain size by the PECVD technique at low temperatures.

It was observed that the fundamental parameters to induce large grain size are the $\mathrm{B}_{2} \mathrm{~N}_{6} / \mathrm{N}_{2}$ flow ratio and low rf power $[12,13]$. In the literature it is observed that the bulk values of the mechanical constants such as hardness, elastic modulus and thermal expansion coefficient, of the h-BN crystals oriented with the c-axis 
perpendicular to the substrate surface [6] are very close to the exhibited by our hexagonal BN samples.

The values obtained for the hardness, the elastic modulus and the thermal expansion coefficient are comparable with those reported for h-BN. The hardness, and stress present opposite behavior with the substrate temperature, the hardness exhibits a maximum and the internal stress a minimum around $180{ }^{\circ} \mathrm{C}$. At this point more study is necessary to understand this behavior, but it is possible to get a hardest h-BN thin film at low temperatures.

\section{Conclusions}

Boron nitride thin films with different structure were obtained by the PECVD technique. The fraction of hexagonal and amorphous phases in the thin films is extremely dependent on the $\mathrm{B}_{2} \mathrm{H}_{6}$ flow and $\mathrm{H}_{2}$ dilution. The crystallite size for the hexagonal grains increases for increasing temperatures and $\mathrm{B}_{2} \mathrm{H}_{6}$ flow as well as for low rf power density. The measured thermo mechanical properties of the BN samples did not show serious variations with the deposition conditions. In addition, the hexagonal BN nano-crystals present in the samples are well oriented and of good size.

\section{Acknowledgments}

The authors are grateful to Drs. M. Tabacknis, F.L. Freire for the RBS and ERDA measurements, and Dr. M. Temperini, Dra. Nelia Ferreira, and A.C. Costa for Raman and Visible equipment facilities. This work was supported by Brazilian agency FAPESP, proc \# 98/01766-5.

\section{References}

[1] C. Rohr, J.-H. Boo, and W. Ho, Thin Solid Films, 9, 322 (1998).
[2] M. Hubacek, M. Ucki, T. Sato, and V. Brozek, Thermochimica Acta, 359, 282\&283 (1996).

[3] T. Wittkowski, J. Jorzick, K. Jung, and B. Hillerbrands, Thin Solid Films, 137, 353 (1999).

[4] J.-L. Huang, Ch-H. Pan, and D.-F. Lii, Surface and Coatings Tech. 166, 122 (1999).

[5] V. L. Solozhenko, D.Hausermann, M. Mezouar, and M. Kunz, App. Phys. Lett. 1691, 72 (14), (1998).

[6] Properties of Group III Nitrides, edited by James H. Edgar, Kansas State University, 1994, published by INSPEC, the Institution of Electrical Engineers, London, United Kingdom.

[7] Y. Kimura, T. Wakabayashi, K. Okada, T. Wada, and H. Nishikawa, Wear, 199, 232 (1999).

[8] R. Riedel, Adv. Mater. 6, 549 (1994).

[9] R.T. Paine and C.K. Narula, Chem. Rev. 90, 73 (1990).

[10] E. Yamaguchi, Mater. Sci. Forum 54-55, 329 (1990).

[11] Synthesis and Properties of Boron Nitride, edited by J.J. Pouch and S.A Alterovitz (Trans. Tech., Zurich, 1990).

[12] M.N.P. Carreño, J.P. Bottechia, and I. Pereyra, Thin Solid Films, 308-309, 219-222 (1997).

[13] J. Vilcarromero, M.N.P. Carreño, and I. Pereyra. Thin Solids Films, 373, (1-2) 273-276 SEP 32000.

[14] R.W. Hoffman, in Physics on thin films, edited by G. Hass and R.E. Thun (Academic, New York, 1966), vol 3 .

[15] J. F. Moulder, W. F. Stickle, P. E. Sobol, and K. D. Bombem, Handbook of X-ray Photoeletron Spectroscopy, edited by J. Chastain, Perkin Elmer Co., 1992.

[16] D. M. Hoffman, G.L. Doll, and P.C. Eklund, Phys. Rev. B 6051, 30 (10), (1984).

[17] R.J. Nemanich, S.A. Solin, and R.M. Martin, Phys. Rev. B (23) 12, 6348, (1981). 